\title{
Impact of sleep quality on clinical features of primary Sjögren's syndrome
}

Sang Wan Chung ${ }^{1,}$, Jaehyung Hur ${ }^{2,}$, You-Jung Ha ${ }^{2}$, Eun Ha Kang ${ }^{2}$, Joon Young Hyon ${ }^{3}$, Hyo-Jung Lee ${ }^{4}$, Yeong Wook Song ${ }^{5,6}$, and Yun Jong Lee ${ }^{2,5}$

${ }^{1}$ Department of Internal Medicine, Graduate School, Kyung Hee University, Seoul; Departments of ${ }^{2}$ Internal Medicine, ${ }^{3}$ Ophthalmology, ${ }^{4}$ Department of Periodontology, Section of Dentistry, Seoul National University Bundang Hospital, Seongnam; ${ }^{5}$ Department of Internal Medicine, ${ }^{6}$ WCU Department of Molecular Medicine and Biopharmaceutical Sciences, Medical Research Institute, Seoul National University College of Medicine, Seoul, Korea

Received: April 13, 2017

Revised : August 22, 2017

Accepted: November 10, 2017

\section{Correspondence to}

Yun Jong Lee, M.D.

Department of Internal Medicine, Seoul National University Bundang Hospital, 82 Gumi-ro 173beon-gil, Bundang-gu, Seongnam 13620, Korea

Tel: $+82-31-787-7049$

Fax: $+82-31-787-4051$

E-mail:yn35@snu.ac.kr

*'These authors contributed equally to this work.
Background/Aims: This study aimed to investigate the influence of poor sleep quality on clinical features of primary Sjögren's syndrome (pSS).

Methods: Sleep quality was cross-sectionally assessed using the Pittsburgh Sleep Quality Index (PSQI), and demographic, clinical, and laboratory data were collected from 115 Korean patients with pSS. The patients completed questionnaires on the European League Against Rheumatism (EULAR) SS Patient Reported Index (ESSPRI), quality of life (EuroQOL five dimensions questionnaire [EQ-5D]), fatigue (fatigue severity score [FSS]), and depression (Beck Depression Inventory [BDI] II]). Symptoms and patient global assessment (PGA) were evaluated with a 100-mm visual analogue scale (VAS). The EULAR sicca score (ESS), ESSPRI, and EULAR SS Disease Activity Index (ESSDAI) were calculated at study enrollment.

Results: Fifty-three patients (46.1\%) had poor sleep quality and $32.4 \%$ of 71 patients without depression were poor sleepers. Poor sleepers had a significantly lower EQ-5D or ESSDAI and a significantly higher FSS, BDI-II, PGA, ESS, ESSPRI, or VAS scores for extra-glandular symptoms than good sleepers. Neutrophil and lymphocyte counts were significantly higher and immunoglobulin G levels tended to decrease in poor sleepers. Additionally, PSQI was negatively correlated with EQ-5D and ESSDAI and positively with ESS, FSS, BDI-II, PGA, VAS scores for their symptoms, and ESSPRI. Multivariate analysis revealed that poor sleep quality remained the independent determinants of the unsatisfactory symptom state (ESSPRI $\geq 5$ ).

Conclusions: Our results showed that poor sleep quality could significantly affect the patient-oriented outcomes and physician-reported activity index of pSS patients through the various effects of sleep quality on the psychological or somatic symptoms and the immune system.

Keywords: Sjogren's syndrome; Sleep hygiene; Patient outcome assessment; Severity of illness index

\section{INTRODUCTION}

Primary Sjögren's syndrome (pSS) is a chronic autoimmune inflammatory disease primarily targeting the exocrine gland and is characterized by the presence of organ specific or non-specific auto-antibodies and focal lymphocytic infiltration in the exocrine glands [1]. Although the major clinical manifestation of pSS is sicca symptoms, $20 \%$ to $40 \%$ of the patients develop wide spectrum of extra-glandular involvement [2]. Such het- 
erogeneous clinical features of pSS can be divided into two groups: benign subjective manifestations, such as dry eye and mouth, myalgia, or fatigue, and systemic manifestations, including vasculitis, renal involvement, and peripheral or central nerve involvement [2].
The European League Against Rheumatism (EULAR) SS Patient Reported Index (ESSPRI) and the EULAR SS Disease Activity Index (ESSDAI) are the tools used to assess the disease activity of SS and are considered the gold-standard outcome measures [3]. ESSPRI is

Table 1. Clinical characteristics of the study subjects

\begin{tabular}{|c|c|c|c|c|}
\hline Characteristic & Total $(\mathrm{n}=115)$ & Good sleeper $(n=62)$ & Poor sleeper $(n=53)$ & $p$ value \\
\hline Female sex & $112(97.4)$ & $60(96.8)$ & $52(98.1)$ & 1.000 \\
\hline Age, yr & $54.0(45.0-63.0)$ & $51.5(44.0-57.5)$ & $59.0(47 \cdot 0-64.5)$ & 0.042 \\
\hline Menopause & $74 / 112(66.1)$ & $36 / 60(60.0)$ & $38 / 52(73.1)$ & 0.145 \\
\hline Symptom duration, yr & $10.0(7.0-15.0)$ & $10.0(7.0-15.0)$ & $12.0(8.0-20.0)$ & 0.073 \\
\hline $\mathrm{WBC}, / \mathrm{mm}^{3}$ & $4,370(3,790-5,450)$ & $4,065(3,515-4,695)$ & $4,660(4,190-5,930)$ & $4.63 \times 10^{-4}$ \\
\hline Neutrophils, $/ \mathrm{mm}^{3}$ & $2,410(1,740-3,179)$ & $2,135(1,563-2,857)$ & $2,513(1,967-3,924)$ & 0.004 \\
\hline Lymphocytes, $/ \mathrm{mm}^{3}$ & $1,589(1,246-1,901)$ & $1,517(1,176-1,711)$ & $1,629\left(1,295^{-2,049)}\right.$ & 0.035 \\
\hline Hemoglobin, g/dL & $12.7(12.0-13.4)$ & $12.6(11.9-13.4)$ & $12.8(12.1-13.4)$ & 0.561 \\
\hline Platelets, $\times 10^{3} / \mathrm{mm}^{3}$ & $220(182-260)$ & $209(182-240)$ & $228(183-271)$ & 0.121 \\
\hline $\mathrm{ESR}, \mathrm{mm} / \mathrm{hr}$ & $21(13-33)$ & $21(13-37)$ & $20(10-31)$ & 0.316 \\
\hline $\mathrm{CRP}, \mathrm{mg} / \mathrm{dL}$ & $0.07(0.03-0.15)$ & $0.06(0.03-0.14)$ & $0.07(0.04-0.17)$ & 0.181 \\
\hline$\beta 2-\mathrm{MG}, \mathrm{mg} / \mathrm{dL}$ & $2.27(1.84-2.64)$ & $2.33(1.87-2.55)$ & $2.10(1.77-2.74)$ & 0.873 \\
\hline IgG, g/dL & $1.71(1.48-2.0)$ & $1.72(1.58-2.06)$ & $1.65(1.38-1.88)$ & 0.061 \\
\hline $\mathrm{C}_{3}, \mathrm{mg} / \mathrm{dL}$ & $99.8(86.3-111.3)$ & $96.5(86.3-107.5)$ & $102.0(86.4-25.2)$ & 0.249 \\
\hline $\mathrm{C}_{4}, \mathrm{mg} / \mathrm{dL}$ & $21.2(18.1-24.7)$ & $19.5(16.5-23.8)$ & $22.2(18.9-25.2)$ & 0.027 \\
\hline ESSPRI & $5.0(3.7-6.7)$ & $4.2(3.0-5.7)$ & $6.0(4.5-7.3)$ & $4.43 \times 10^{-4}$ \\
\hline ESSPRI $\geq 5$ & $62(53.9)$ & $24(38.7)$ & $38(61.3)$ & $4.04 \times 10^{-4}$ \\
\hline ESSDAI & $2(1-4)$ & $2.5(1-4)$ & $1(0-3)$ & 0.011 \\
\hline $\mathrm{ESSDAI} \geq 5$ & $19(16.5)$ & $12(19.4)$ & $7(13.2)$ & 0.376 \\
\hline \multicolumn{5}{|l|}{ Current medications } \\
\hline Pilocarpine & $59(51.3)$ & $25(40.3)$ & $34(64.2)$ & 0.011 \\
\hline HCQ & $30(26.1)$ & $19(30.6)$ & $11(20.8)$ & 0.229 \\
\hline PD & $24(20.9)$ & $10(16.1)$ & $14(26.4)$ & 0.175 \\
\hline PD dose, mg/day & $0.0(0.0-0.0)$ & $0.0(0.0-0.0)$ & $0.0(0.0-2.5)$ & 0.160 \\
\hline Azathioprine & $2(1.7)$ & $1(1.6)$ & $1(1.9)$ & 1.000 \\
\hline Benzodiazepines & $13(11.3)$ & $4(6.5)$ & $9(17.1)$ & 0.086 \\
\hline Hypnotics & $8(6.1)$ & $1(1.6)$ & $7(13.2)$ & 0.023 \\
\hline SSRI & $5(4 \cdot 3)$ & $1(1.6)$ & $4(7 \cdot 5)$ & 0.079 \\
\hline SNRI & $3(2.6)$ & $1(1.8)$ & $2(3.8)$ & 0.594 \\
\hline TCA & $3(2.9)$ & 0 & $3(5.7)$ & 0.095 \\
\hline
\end{tabular}

Values are presented as number (\%) or median (interquartile range). $p$ values by the Mann-Whitney test, chi-square, or Fisher exact test.

WBC, white blood cell; ESR, erythrocyte sedimentation rate; CRP, C-reactive protein; $\beta 2-\mathrm{MG}, \beta 2$-microglobulin; IgG, immunoglobulin G; ESSPRI, European League Against Rheumatism (EULAR) Sjögren's syndrome (SS) Patient Reported Index; ESSDAI, EULAR SS Disease Activity Index; HCQ, hydroxychloroquine; PD, prednisolone; SSRI, selective serotonin reuptake inhibitor; SNRI, serotonin-norepinephrine reuptake inhibitor; TCA, tricyclic antidepressant. 
used to evaluate the subjective perception of the most important and frequent symptoms, and ESSDAI measures 12 organ-specific domains based on the severity of extra-glandular involvement $[4,5]$. The ESSPRI and ESSDAI, which are not correlated, reflect two different facets (benign sicca symptoms and severe systemic manifestations) of the clinical disease [2].

Increased sleep disturbance has been reported in patients with several rheumatic diseases, such as rheumatoid arthritis (RA) and systemic lupus erythematosus $[6,7]$. Sleep disturbance could result from disease-associated inflammatory symptoms and depression [8]. Sleep disturbance could affect the immune and inflammatory response and may increase the risk of developing an autoimmune disease [9]. As previously reported, the prevalence of sleep disturbance in patients with pSS was significantly higher compared to controls or to patients with RA [10-12], and poor sleep quality in pSS was associated with pain, fatigue, and depression $[10,13,14]$. These results suggest an association between sleep disturbance and the disease activity of pSS. However, no studies on their association in patients with pSS have been published. Thus, we studied the prevalence of poor sleepers and investigated the association between sleep quality and the disease activity indices in Korean patients with pSS.

\section{METHODS}

\section{Study subjects}

We consecutively enrolled 115 patients with pSS (112 females and three males; mean age, 53.0 \pm 1.2 years), who had been followed in our Rheumatology Clinic from January 2015 to April 2016. All patients satisfied the classification criteria of the American European Consensus Group for pSS [15]. The demographic, clinical, and laboratory variables of the patients, including medications prescribed over 3 months, were collected. At enrollment, the ESSPRI, EULAR sicca score (ESS), and ESSDAI were

Table 2. Patient-reported indices of the study subjects

\begin{tabular}{|c|c|c|c|}
\hline Variable & Good sleeper $(n=62)$ & Poor sleeper $(\mathrm{n}=53)$ & $p$ value \\
\hline EQ-5D score & $0.79(0.70-1.00)$ & $0.69(0.62-0.75)$ & $2.14 \times 10^{-4}$ \\
\hline Overall oral dryness VAS & $46.0(9.5-73.0)$ & $60.0(22.5-80.0)$ & 0.128 \\
\hline Nocturnal oral dryness VAS & $20.0(6.0-62.5)$ & $40.0(5.0-70.0)$ & 0.757 \\
\hline XI score & $34(25-45)$ & $41(29-47)$ & 0.098 \\
\hline Overall eye dryness VAS & $40.0(8.0-69.3)$ & $60.0(22.2-80.0)$ & 0.044 \\
\hline Eyeball pain VAS & $10.0(0-40)$ & $20.0(2.0-70.0)$ & 0.176 \\
\hline OSDI score & $20.8(10.4-31.3)$ & $23.0(13.5-41.7)$ & 0.100 \\
\hline ESS & $5.0(2.9-5.7)$ & $6.0(5.0-7 \cdot 3)$ & 0.013 \\
\hline Arthralgia VAS & $10.0(0.0-28.0)$ & $40(4 \cdot 5-60.0)$ & 0.005 \\
\hline Myalgia VAS & $10.0(0.0-29.0)$ & $20.0(1.0-60.0)$ & 0.055 \\
\hline Anxiety VAS & $2.5(0.0-20.0)$ & $10.0(0.0-54.0)$ & 0.037 \\
\hline PGA VAS & $10.0(2.8-40.0)$ & $49.0(20.0-70.0)$ & $4.99 \times 10^{-4}$ \\
\hline PSQI score & $6.0(3.0-7.3)$ & $12.0(10.0-15.0)$ & $2.31 \times 10^{-20}$ \\
\hline FSS score & $2.56(1.78-4.50)$ & $4.56(3.06-5.78)$ & $2.64 \times 10^{-4}$ \\
\hline FSS score $\geq 4$ & $19(30.6)$ & $30(56.6)$ & 0.005 \\
\hline BDI-II score & $7 \cdot 5(4.0-12.3)$ & $15.0(7.5-21.5)$ & $6.42 \times 10^{-5}$ \\
\hline BDI-II score $\geq 14$ & $14(22.6)$ & $30(56.6)$ & $1.83 \times 10^{-4}$ \\
\hline
\end{tabular}

Values are presented as median (interquartile range) or number (\%). p values by the Mann-Whitney or chi-square test.

EQ-5D, EuroQol 5-dimensional questionnaire; VAS, visual analogue scale; XI, xerostomia inventory; OSDI, Ocular Surface Disease Index; ESS, European League Against Rheumatism (EULAR) sicca score; PGA, patient global assessment for Sjögren's syndrome (SS); PSQI, Pittsburgh Sleep Quality Index; FSS, fatigue severity score; BDI, Beck Depression Inventory. 
calculated as previously defined $[4,5]$. A moderately active disease was defined as an ESSDAI $\geq 5$ and an unsatisfactory symptom state was defined as an ESSPRI $\geq 5[16]$. This study was approved by our Institutional Review Board (IRB No. B-0506/021-004) and written informed consent was obtained from all participants.

\section{The questionnaires}

The patients completed the Korean version of the Pittsburgh Sleep Quality index (PSQI) [17]. The PSQI is a self-reported questionnaire to discriminate between "good" and "poor" sleepers and widely used for evalu-

Table 3. Correlations between Pittsburgh Sleep Quality Index and clinical variables

\begin{tabular}{|c|c|c|}
\hline Variable & $\begin{array}{l}\text { Spearman's } \\
\text { coefficient }\end{array}$ & $p$ value \\
\hline Age & 0.189 & 0.043 \\
\hline EQ-5D score & -0.418 & $3.22 \times 10^{-6}$ \\
\hline Overall oral dryness VAS & 0.214 & 0.022 \\
\hline XI score & 0.206 & 0.027 \\
\hline Overall eye dryness VAS & 0.246 & 0.009 \\
\hline Eyeball pain VAS & 0.192 & 0.043 \\
\hline OSDI score & 0.246 & 0.008 \\
\hline ESS & 0.288 & 0.002 \\
\hline Arthralgia VAS & 0.312 & 0.001 \\
\hline Myalgia VAS & 0.300 & 0.001 \\
\hline Anxiety VAS & 0.278 & 0.003 \\
\hline PGA VAS & 0.366 & $5.87 \times 10^{-5}$ \\
\hline FSS score & 0.402 & $8.58 \times 10^{-6}$ \\
\hline BDI-II score & 0.507 & $7.34 \times 10^{-9}$ \\
\hline ESSPRI & 0.432 & $1.44 \times 10^{-6}$ \\
\hline WBC counts & 0.236 & 0.011 \\
\hline ANC & 0.234 & 0.012 \\
\hline IgG levels & -0.218 & 0.019 \\
\hline ESSDAI & -0.259 & 0.005 \\
\hline
\end{tabular}

$p$ values by the Spearman rank correlation test.

EQ-5D, EuroQol 5-dimensional questionnaire; VAS, visual analogue scale; XI, xerostomia inventory; OSDI, Ocular Surface Disease Index; ESS, European League Against Rheumatism (EULAR) sicca score; PGA, patient global assessment for Sjögren's syndrome (SS); FSS, fatigue severity score; BDI, Beck Depression Inventory; ESSPRI, EULAR SS Patient Reported Index; WBC, white blood cell; ANC, absolute neutrophil counts; IgG, immunoglobulin G; ESSDAI, EULAR SS Disease Activity Index. ating sleep quality because of its broad range in measuring several aspects of sleep quality and combining results into a global score [18]. Based on the published literature, a cut-off total score of 8.5 was used to identify Korean poor sleepers in this study [17]. All patients answered the Korean version of questionnaires on quality of life (EuroQol 5-dimensional questionnaire [EQ-5D]), fatigue (fatigue severity score $[\mathrm{FSS}]$ ), and depression (Beck Depression Inventory [BDI] II) [19-21]. Depression was defined as a BDI-II score $\geq 14$, and we used $\geq 4.0$ as the cut-off value for the FSS to define the presence of fatigue as suggested by Valko et al. [22]. Moreover, to evaluate the symptom severity, we used the xerostomia inventory (XI), the Ocular Surface Disease Index (OSDI), and a $10-\mathrm{cm}$, visual analogue scale (VAS) for overall oral dryness, nocturnal oral dryness, overall eye dryness, eyeball pain, arthralgia, myalgia, and anxiety $[23,24]$. We also measured the patient global assessment (PGA) using a VAS.

\section{Statistical analysis}

Continuous values were expressed as the median (interquartile range [IQR]) because a normal distribution was not observed in most variables. The Mann-Whitney test or Kruskal-Wallis test was used to compare the continuous variables. We performed the Jonckheere-Terpstra test to check for trends and used the chi-square or Fisher exact test to compare categorical variables. Spearman's rank correlation coefficient was calculated by bivariate correlation. To find the independent factors of poor sleep quality or the unsatisfactory symptom state (ESSPRI $\geq 5$ ), we performed a stepwise multivariate logistic regression analysis, using a forward selection method. All variables with $p<0.1$ in the univariate analyses were included and all continuous variables except age were converted into categorical variables in the multivariate analysis. Statistical analysis was performed with SPSS version 22 (IBM Co., Armonk, NY, USA). A p 0.05 was considered significant.

\section{RESULTS}

\section{Patient characteristics and the prevalence of poor sleep quality in pSS patients}

The characteristics of the patients with pSS are sum- 
marized in Table 1. Sixty-two patients (53.9\%) had an unsatisfactory symptom state and $19(16.5 \%)$ had a moderate-to-high disease activity (ESSDAI $\geq 5$ ). The median total PSQI score was 8.0 (IQR, 5.0 to 12.0; mean \pm stan- dard deviation, $8.92 \pm 4.62$ ) and the prevalence of poor sleepers (PSQI > 8.5) was $46.1 \%$.

When patients with pSS were classified into poor and good sleepers according to their PSQI scores, the poor
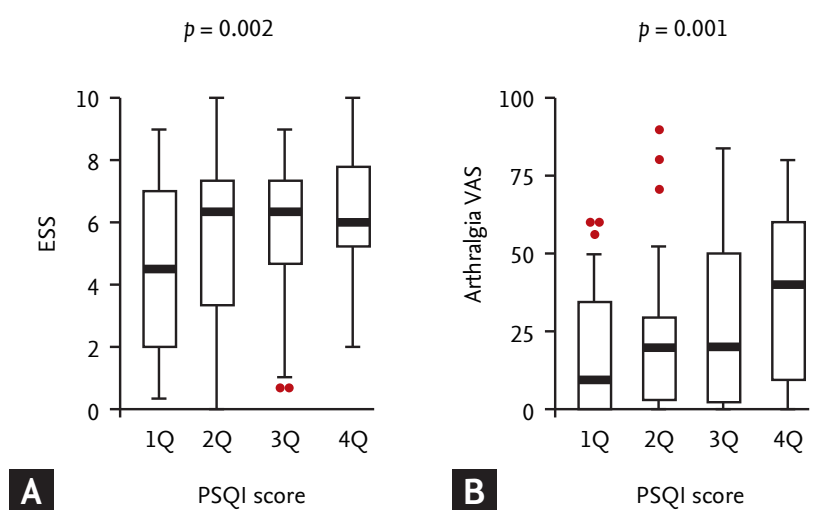

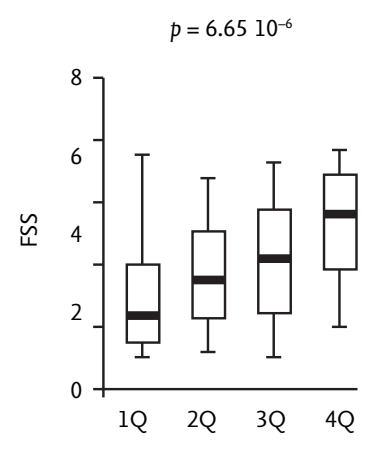

C

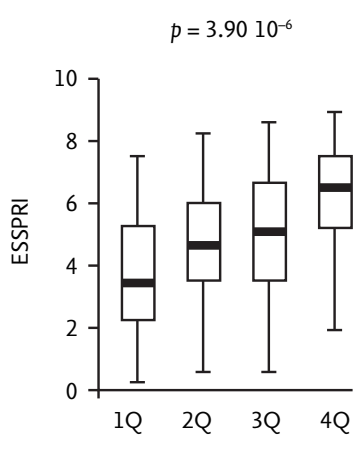

D PSQI score

Figure 1. Distribution of patient-reported measures according to the Pittsburgh Sleep Quality Index (PSQI) quartiles. The distribution of (A) EULAR sicca score (ESS) ( $p=0.018$ by the Kruskal-Wallis test), (B) arthralgia visual analogue scale (VAS) scores $(p=0.013),(C)$ fatigue severity score $(F S S)\left(p=1.82 \times 10^{-4}\right)$, and (D) EULAR SS Patient Reported Index $($ ESSPRI $)\left(p=6.95 \times 10^{-5}\right)$ was significantly different among the PSQI quartiles. Additionally, a significant positive trend for the ESS, arthralgia VAS, and FSS were found across the PSQI quartiles. Eventually, a significant positive trend of the ESSPRI was observed across the PSQI quartiles. $p$ values above the figures were calculated by the Jonckheere-Terpstra test. EULAR, European League Against Rheumatism; SS, Sjögren's syndrome.

Table 4. Multivariate analysis by logistic regression

\begin{tabular}{|c|c|c|c|c|}
\hline & $\beta$ & $\mathrm{SE}$ & $p$ value & OR (95\% CI) \\
\hline \multicolumn{5}{|c|}{ Poor sleep quality (PSQI $\geq 8.5$ ) as a dependent variable } \\
\hline \multicolumn{5}{|l|}{ All subjects $(\mathrm{n}=115)$} \\
\hline Depression (BDI-II $\geq 14)$ & 1.618 & 0.469 & 0.001 & $5.043(2.012-12.640)$ \\
\hline Leukopenia & -1.475 & 0.512 & 0.004 & $0.229(0.084-0.625)$ \\
\hline Pilocarpine usage & 1.031 & 0.450 & 0.022 & $2.804(1.160-6.779)$ \\
\hline \multicolumn{5}{|l|}{ Subjects without depression $(n=71)$} \\
\hline Unsatisfactory symptom state (ESSPRI $\geq 5$ ) & 1.505 & 0.586 & 0.010 & $4.505(1.428-14.214)$ \\
\hline Ever-smoking & 1.988 & 0.976 & 0.042 & $7.298(1.078-49.422)$ \\
\hline \multicolumn{5}{|l|}{ Subjects with depression $(\mathrm{n}=44)$} \\
\hline Leukopenia & -3.068 & 1.111 & 0.006 & $0.047(0.005-0.410)$ \\
\hline Age & 0.120 & 0.050 & 0.017 & $1.128(1.021-1.245)$ \\
\hline \multicolumn{5}{|c|}{ Unsatisfactory symptom state (ESSPRI $\geq 5$ ) as a dependent variable } \\
\hline Fatigue $(\mathrm{FSS} \geq 4)$ & 2.393 & 0.542 & $1.00 \times 10^{-5}$ & $10.942(3.784-31.635)$ \\
\hline PGA, the 4 th quartile & 2.011 & 0.772 & 0.009 & $7.469(1.645-33.915)$ \\
\hline Poor sleep quality & 1.162 & 0.513 & 0.023 & $3.197(1.171-8.731)$ \\
\hline PD usage & 1.388 & 0.657 & 0.035 & $4.006(1.105-14.526)$ \\
\hline
\end{tabular}

SE, standard error; OR, odds ratio; CI, confidence interval; PSQI, Pittsburgh Sleep Quality Index; BDI, Beck Depression Inventory; ESSPRI, European League Against Rheumatism (EULAR) Sjögren's syndrome (SS) Patient Reported Index; FSS, fatigue severity score; PGA, patient global assessment for SS; PD, prednisolone. 
$p=0.018$

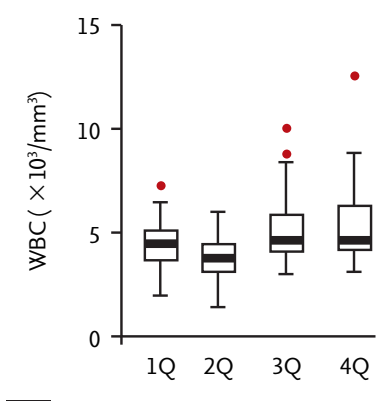

A
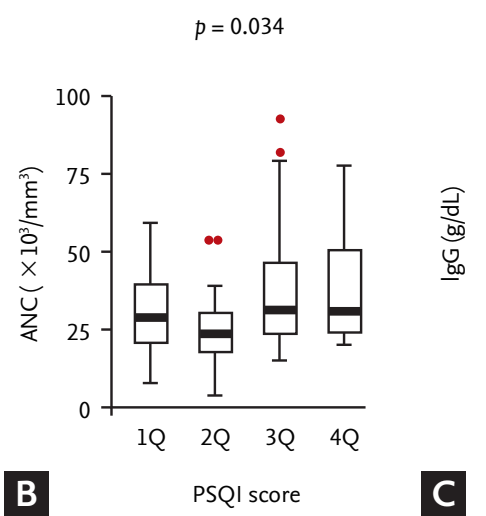

$p=0.029$

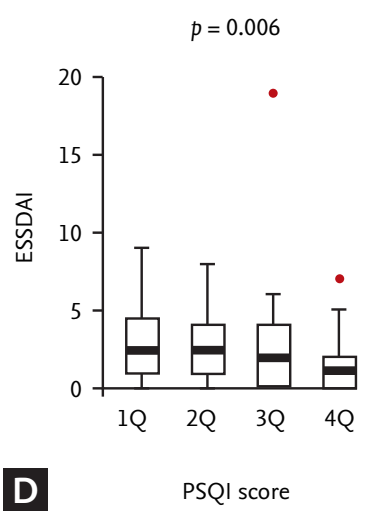

Figure 2. Distribution of the hematological and immunological parameters according to the Pittsburgh Sleep Quality Index (PSQI) quartiles. The distribution of the (A) white blood cell (WBC) counts ( $p=0.001$ by the Kruskal-Wallis test) and (B) absolute neutrophil counts (ANC; $p=0.013$ ) was significantly different among the PSQI quartiles, even though the difference in the (C) immunoglobulin G (IgG) levels $(p=0.154)$ was not significant. Additionally, a significant positive trend for the WBC counts or ANC and a negative trend for the IgG levels were found across the PSQI quartiles. (D) Eventually, the distribution of the EULAR SS Disease Activity Index (ESSDAI) was significantly different $(p=0.037)$ and a significant negative trend for the ESSDAI was observed across the PSQI quartiles. $p$ values above the figures were calculated by the Jonckheere-Terpstra test. EULAR, European League Against Rheumatism; SS, Sjögren's syndrome.

sleepers were significantly older (median age, 59 years vs. 52 years, $p<0.05)$. The prevalence for the fulfilled classification criteria at the time of diagnosis was not different, except for a minor salivary gland biopsy with a focus score $\geq 1$ (100\% in poor sleepers vs. $68.2 \%$ in good sleepers, $p<0.001)$. Poor sleepers were more likely to take pilocarpine $(64.2 \%$ vs. $40.3 \%, p<0.05)$ and hypnotics (13.2\% vs. $1.6 \%, p<0.05)$. However, the frequency of comorbidities including fibromyalgia $(n=7)$ and restless leg syndrome $(n=7)$ was comparable between the two subgroups.

At enrollment, poor sleepers exhibited a significantly lower prevalence of leukopenia ( $17.0 \%$ vs. $48.4 \%, p<$ 0.001 ) and neutropenia (5.7\% vs. 22.6\%, $p<0.05)$. Concordantly ESSDAI was significantly lower in poor sleeper than in good sleepers (median, 1 vs. 2.5, $p<0.05$ ) although the proportion of patients with moderate-to-high disease activity was not different between the two subgroups.

\section{Comparison of ESSPRI and other patient-reported indices between good and poor sleepers}

Concerning self-reported symptom severity, poor sleepers had a significantly higher VAS level of overall eye dryness (median, 60 vs. 40, $p<0.05$ ), arthralgia (40 vs. 10, $p<0.01$ ), anxiety (10 vs. $2.5, p<0.05$ ), and overall PGA (49 vs. 10, $p<0.001$ ) compared to the good sleepers (Table 2). The XI, OSDI, and VAS scores for overall oral dryness, night oral dryness, eyeball pain, and myalgia tended to be higher in poor sleeper; however, the difference was not significant. Additionally, the EQ-5D score was significantly lower (median, 0.690 vs. $0.785, p<0.001$ ) and the $\operatorname{ESS}(6.0$ vs. $5.0, p<0.05$ ), FSS ( 41 vs. $23, p<0.001$ ), and BDI-II (15 vs. 7.5, $p<1 \times 10^{-4}$ ) scores were significantly higher in poor sleepers than in good sleepers (Table 2). Thirty of $44 \mathrm{pSS}$ patients (68.2\%) with depression reported poor sleep quality, whereas 23 of 71 patients (32.4\%) without depression were poor sleepers $(p<0.001)$. Poor sleepers showed a significantly higher ESSPRI compared to good sleepers (median, 6.0 vs. 4.2, $p<0.001$ ), and the proportion of subjects with a patient-unacceptable symptom state was $61.3 \%$ in poor sleepers and $38.7 \%$ in good sleepers $(p<0.001)$.

\section{Correlations between sleep quality and clinical variables}

From the bivariate correlation analyses, age and EQ-5D were significantly positively and negatively associated with the PSQI scores, respectively (Table 3). The PSQI score was a significant determinant of the highest EQ${ }_{5} \mathrm{D}$ quartile in the multivariate analysis $(p=0.013$; odds ratio, 1.165; 95\% confidence interval, 1.032 to 1.315). 
The XI, OSDI, and VAS scores for sicca symptoms, eyeball pain, arthralgia, myalgia, and anxiety were significantly associated with the PSQI scores in all patients with pSS (all $p<0.05)$ (Table 3$)$. The PSQI scores were significantly positively correlated with the BDI-II and FSS scores $\left(\right.$ all $\left.p<1 \times 10^{-5}\right)$ and the white blood cell (WBC) or absolute neutrophil counts (ANC), but negatively correlated with the total immunoglobulin $\mathrm{G}$ (IgG) levels (all $p<0.05$ ) (Table 3). Moreover, the PSQI was positively associated with the ESSPRI $\left(p<1.0 \times 10^{-5}\right)$ and negatively associated with the $\operatorname{ESSDAI}(p=0.005)$.

When the patients were stratified according to PSQI quartiles, the distribution of ESS, arthralgia VAS, FSS, WBC counts or ANC, ESSPRI and ESSDAI was significantly different across the quartiles (all $p<0.05$ ) (Figs. 1 and 2). In addition, a significant trend was found across the PSQI quartiles for ESS, arthralgia VAS, FSS, WBC counts, ANC, and serum IgG (all $p<0.05$ ) (Figs. 1 and 2). Finally, significant increasing trends of ESSPRI $(p<$ $\left.1.0 \times 10^{-5}\right)$ or decreasing trends of ESSDAI $(p<0.01)$ were observed across the increasing quartiles. However, a significant trend for the ESSDAI was not observed when patients were stratified according to quartiles of FSS, BDI-II, or other patient symptom severity levels.

\section{Independent association between poor sleep quality and ESSPRI in pSS patients}

Because the variables were mutually correlated, a multivariate analysis was performed adjusting for age to identify factors associated with poor sleep quality in patients with pSS. All continuous variables except for age were entered as dichotomous variables based on the highest or lowest quartile or clinically relevant cut-points (ES$\mathrm{SPRI} \geq 5, \mathrm{ESSDAI} \geq 5, \mathrm{FSS} \geq 4$, BDI-II $\geq 14$, leukopenia $[\mathrm{WBC}<4,000 / \mu \mathrm{L}]$, or neutropenia $[\mathrm{ANC}<1,500 / \mu \mathrm{L}]$ ) [16,17,20-22].

Poor sleep quality was significantly independently associated with depression, leukopenia, and pilocarpine usage in all patients with pSS (Table 4). Although poor sleep quality is strongly associated with depression, about a third of the pSS patients without depression had poor sleep quality in this study. Therefore, multivariate analysis was repeated after dividing the study subjects according to a BDI-II $\geq 14$. Poor sleep quality was significantly associated with the unsatisfactory symptom state (ESSPRI $\geq 5)$ and ever-smoking in pSS patients with- out depression. Furthermore, in a multivariate analysis with the ESSPRI $\geq 5$ as the dependent variable, poor sleep quality was an independent contributor to the patient-unacceptable symptom state.

Univariate analyses revealed that poor sleep quality was significantly associated with the WBC or neutrophil counts and IgG levels, and multivariate analysis showed a negative association with leukopenia. Although these variables are the components included in calculating the ESSDAI, an independent association between poor sleep quality and ESSDAI was not observed in the multivariate analysis.

\section{DISCUSSION}

This study showed that $46.1 \%$ of the patients with pSS reported poor sleep quality and, even among the pSS patients without depression, 32.4\% were poor sleepers. These poor sleepers had more severe glandular and extra-glandular symptoms compared to the good sleepers. Furthermore, for the first time, we found that poor sleep quality in patients with pSS was independently associated with the ESSPRI and that the ESSDAI might be underestimated in poor sleepers, because poor sleep quality could be negatively associated with the WBC or neutrophil counts and IgG levels.

Sleep impairment is common in chronic inflammatory diseases [8]. However, the prevalence of sleep disturbance in patients with pSS has not been extensively studied. Nevertheless, in a previous study, $75 \%$ of 65 Israeli patients with pSS had moderate to severe sleep disturbance based on the Mini Sleep Questionnaire [10]. In addition, in 40 Swedish patients with pSS, 72\% complained of "too little sleep," and in 29 Italian patients with pSS, $82.8 \%$ had sleep complaints (mean PSQI score, $8.62 \pm 4.58)[11,13]$. In contrast, Segal et al. [12] reported that $37.8 \%$ of 108 American patients with pSS had self-reported sleep disorder. Such variation may be related to different assessment methods or to the different prevalence of depression in the study subjects. Moreover, previous studies have shown that the prevalence of disturbed sleep was significantly higher in patients with pSS than in patients with RA or in controls [10,11,13]. Although a control group was not included in this study, the PSQI scores of the subjects were much higher than 
those in previous reports with Korean subjects (4.06 \pm 2.08 in 133 healthy controls, $5.62 \pm 4.19$ in 130 RA patients, and $6.0 \pm 3.6$ in 2,040 subjects aged $\geq 60$ years) $[17,25,26]$. These data show that a significant portion of patients with pSS appears to have poor sleep quality to a similar extent as patients with other rheumatic diseases [8].

Whether sleep alterations are the results of disease-related symptoms, including sicca symptoms, pain, and depression, or disease-related immune changes remains unclear. Severe xerostomia may disturb nocturnal sleep because the salivary flow rate decreases during sleep. It was also shown that dry mouth in patients with pSS was independently associated with sleep quality and that subjects complaining of dry mouth had a decrease in sleep quality [27]. Furthermore, poor sleep is common in individuals with dry eye; sleep quality was associated the severity of dry eye [28]. Sleep deprivation could reduce tear secretion and decreases the tear film break-up time [29]. However, in this study, although poor sleepers had a higher ESS than that of the good sleepers, their self-reported level of oral and dry symptoms was not significantly higher, except for overall eye dryness.

We observed that poor sleepers reported a higher level of arthralgia or myalgia in this study. The severity of neuropathic pain was previously shown to be associated with poor sleep quality in patients with pSS [12], but no reports on the association between musculoskeletal pain and sleep disturbance are available in patients with pSS. In contrast, an association between arthralgia and sleep disturbance has been clearly shown in RA patients [6]. Sleep impairments are suggested to be a stronger and reliable predictor of pain, and sleep deprivation can increase RA-related joint pain [30].

The pSS-related fatigue is a major distressing problem of patients with pSS. In this study, pSS patients with poor sleep quality showed a significantly higher level of fatigue. Because of the restorative function of sleep, it has been generally accepted that disturbed sleep is associated with fatigue. The pSS-related fatigue was previously reported to be associated with personality traits and various clinical features, including disturbed sleep $[13,31,32]$. Furthermore, sleep deprivation can increase the expression of interleukin 6, which have play a role in both pSS-associated inflammation and fatigue [9].

Depression, which is frequently accompanied by sleep disturbances, is common in pSS and strongly associat- ed with fatigue, working disability, and widespread pain $[31,32]$. In this study, the prevalence of depression was significantly higher in poor sleepers than in good sleepers, and depression was independently associated with poor sleep quality in the multivariate analysis. However, because a third of the pSS patients without depression were poor sleepers, poor sleep quality is not caused by depression alone. Thus, it can be deduced that poor sleep quality could be affected by the three key symptoms (sicca symptoms, pain, and fatigue) as well as by depression in patients with pSS.

ESSPRI is a disease activity index to incorporate patient-reported measures of the main pSS symptoms and was suggested to be disease-specific [33]. ESSPRI, as a composite measure, could more closely reflect various aspects of sleep than a single clinical measure, considering the complex interaction between sleep and the pSS pathomechanism. In our study, the multivariate analysis showed that poor sleep quality was independently associated with the unsatisfactory symptom state (ESSPRI $\geq$ 5) in patients with pSS, and in those without depression, the unsatisfactory symptom state remained an independent factor for poor sleep quality. Although sialogogues or topical treatment for sicca symptoms led to the reduction of sleep disturbance, no reports on the change in sleep quality after the introduction of treatment targeting ESSPRI in patients with pSS are available, except for rituximab $[34,35]$. Recently, the use of biologic agents and tofacitinib have been reported to improve sleep quality and disease activity in the clinical trials of RA [36]. Therefore, a therapy improving the ESSPRI could improve the sleep quality in patients with pSS.

Another unique finding of this study is the lower ESSDAI level in poor sleepers than in good sleepers. Sleep restriction reportedly increases circulating the number of WBCs, neutrophils, and lymphocytes and reduces antigen-derived B cell response [37,38]. These findings suggest that disturbed sleep could affect the immunopathological or laboratory features of patients with pSS. In this study, the PSQI was positively correlated with the WBC and neutrophil counts and negatively correlated with the serum IgG levels. Poor sleepers exhibited a higher WBC, as well as neutrophil and lymphocyte counts in their peripheral blood compared to good sleepers. Furthermore, the multivariate analysis showed that leukopenia was negatively associated with 
poor sleep quality. Eventually, the interaction between poor sleep quality and circulating neutrophils or IgG levels may reduce the score for the hematological or biological domain of the ESSDAI. Therefore, sleep quality could act as a hidden confounder in the ESSDAI estimation. However, because it failed to remain statistically significant in the multivariate analysis, the association between sleep quality and the ESSDAI needs to be tested in future studies.

This study has several limitations. First, the sample size was not large, and most subjects had a mild systemic disease activity. Thus, further studies with large sample sizes and patients with a higher ESSDAI are warranted for a definite conclusion on the association between sleep quality and disease activity indices. Second, matched controls were not enrolled. However, the total PSQI scores of our Korean patients with pSS were much higher than those of other Korean subjects in previous reports and were comparable to the results in a study by Priori et al. $[13,17,25,26]$. Third, sleep quality was evaluated using only a self-reported questionnaire. Although polysomnography is considered the gold standard for primary sleep disorder assessment, it is labor-consuming and expensive. Moreover, considering the inconsistent association between polysomnographic findings and the PSQI, polysomnography and PSQI could evaluate different aspects of the sleep problem [39]. Lastly, because sleep quality can be influenced by many internal and external factors, all confounding factors could not be excluded in this study. Nonetheless, our results suggest that the ESSPRI is not a simple combination of the three symptoms and have implications in diverse aspects of pSS including sleep quality.

In conclusion, our study showed that poor sleep quality is common in Korean patients with pSS and that an unsatisfactory symptom state is independently associated with poor sleep quality. Furthermore, poor sleep quality may lead to an underestimation of the ESSDAI in pSS. Therefore, clinicians should be aware of the possibility of poor sleep quality in pSS patients with an unsatisfactory symptom state.

\section{KEY MESSAGE}

1. Poor sleep quality is common in Korean pri- mary Sjögren's syndrome (pSS) patients, even in those without depression.

2. Poor sleep quality is an independent determinant of the unsatisfactory symptom state in pSS.

3. Poor sleep quality may lead to an underestimation of physician-administered systemic activity index in pSS.

\section{Conflict of interest}

This work was supported by the Yuhan Corporation (Grant number 06-2016-160).

\section{REFERENCES}

1. Ramos-Casals M, Font J. Primary Sjogren's syndrome: current and emergent aetiopathogenic concepts. Rheumatology (Oxford) 2005;44:1354-1367.

2. Seror R, Theander E, Bootsma H, et al. Outcome measures for primary Sjogren's syndrome: a comprehensive review. J Autoimmun 2014;51:51-56.

3. Seror R, Theander E, Brun JG, et al. Validation of EULAR primary Sjogren's syndrome disease activity (ESSDAI) and patient indexes (ESSPRI). Ann Rheum Dis 2015;74:859866.

4. Seror R, Ravaud P, Mariette X, et al. EULAR Sjogren's Syndrome Patient Reported Index (ESSPRI): development of a consensus patient index for primary Sjogren's syndrome. Ann Rheum Dis 2011;70:968-972.

5. Seror R, Ravaud P, Bowman SJ, et al. EULAR Sjogren's syndrome disease activity index: development of a consensus systemic disease activity index for primary Sjogren's syndrome. Ann Rheum Dis 2010;69:1103-1109.

6. Lee YC, Chibnik LB, Lu B, et al. The relationship between disease activity, sleep, psychiatric distress and pain sensitivity in rheumatoid arthritis: a cross-sectional study. Arthritis Res Ther 2009;11:R160.

7. Palagini L, Tani C, Mauri M, et al. Sleep disorders and systemic lupus erythematosus. Lupus 2014;23:115-123.

8. Abad VC, Sarinas PS, Guilleminault C. Sleep and rheumatologic disorders. Sleep Med Rev 2008;12:211-228.

9. Ranjbaran Z, Keefer L, Stepanski E, Farhadi A, Keshavarzian A. The relevance of sleep abnormalities to chronic inflammatory conditions. Inflamm Res 2007;56:51-57. 
10. Tishler M, Barak Y, Paran D, Yaron M. Sleep disturbances, fibromyalgia and primary Sjogren's syndrome. Clin Exp Rheumatol 1997;15:71-74.

11. Gudbjornsson B, Broman JE, Hetta J, Hallgren R. Sleep disturbances in patients with primary Sjogren's syndrome. Br J Rheumatol 1993;32:1072-1076.

12. Segal BM, Pogatchnik B, Henn L, Rudser K, Sivils KM. Pain severity and neuropathic pain symptoms in primary Sjogren's syndrome: a comparison study of seropositive and seronegative Sjogren's syndrome patients. Arthritis Care Res (Hoboken) 2013;65:1291-1298.

13. Priori R, Minniti A, Antonazzo B, Fusconi M, Valesini G, Curcio G. Sleep quality in patients with primary Sjogren's syndrome. Clin Exp Rheumatol 2016;34:373-379.

14. Theander L, Strombeck B, Mandl T, Theander E. Sleepiness or fatigue? Can we detect treatable causes of tiredness in primary Sjogren's syndrome? Rheumatology (Oxford) 2010;49:1177-1183.

15. Vitali C, Bombardieri S, Jonsson R, et al. Classification criteria for Sjogren's syndrome: a revised version of the European criteria proposed by the American-European Consensus Group. Ann Rheum Dis 2002;61:554-558.

16. Seror R, Bootsma H, Saraux A, et al. Defining disease activity states and clinically meaningful improvement in primary Sjogren's syndrome with EULAR primary Sjogren's syndrome disease activity (ESSDAI) and patient-reported indexes (ESSPRI). Ann Rheum Dis 2016;75:382-389.

17. Sohn SI, Kim DH, Lee MY, Cho YW. The reliability and validity of the Korean version of the Pittsburgh Sleep Quality Index. Sleep Breath 2012;16:803-812.

18. Omachi TA. Measures of sleep in rheumatologic diseases: Epworth Sleepiness Scale (ESS), Functional Outcome of Sleep Questionnaire (FOSQ), Insomnia Severity Index (ISI), and Pittsburgh Sleep Quality Index (PSQI). Arthritis Care Res (Hoboken) 2011;63 Suppl 11:S287-S296.

19. Lee YK, Nam HS, Chuang LH, et al. South Korean time trade-off values for EQ-5D health states: modeling with observed values for 101 health states. Value Health 2009;12:1187-1193.

20. Lee JH, Jeong HS, Lim SM, et al. Reliability and validity of the fatigue severity scale among university student in South Korea. Korean J Biol Psychiatry 2013;20:6-11.

21. Sung HM, Kim JB, Park YN, Bai DS, Lee SH, Ahn HN. A study on the reliability and the validity of Korean version of the Beck Depression Inventory-II (BDI-II). J Korean
Soc Biol Ther Psychiatry 2008;14:201-212.

22. Valko PO, Bassetti CL, Bloch KE, Held U, Baumann CR. Validation of the fatigue severity scale in a Swiss cohort. Sleep 2008;31:1601-1607.

23. Thomson WM, Williams SM. Further testing of the xerostomia inventory. Oral Surg Oral Med Oral Pathol Oral Radiol Endod 2000;89:46-50.

24. Schiffman RM, Christianson MD, Jacobsen G, Hirsch JD, Reis BL. Reliability and validity of the Ocular Surface Disease Index. Arch Ophthalmol 2000;118:615-621.

25. Son CN, Choi G, Lee SY, et al. Sleep quality in rheumatoid arthritis, and its association with disease activity in a Korean population. Korean J Intern Med 2015;30:384-390.

26. Chang KJ, Son SJ, Lee Y, et al. Perceived sleep quality is associated with depression in a Korean elderly population. Arch Gerontol Geriatr 2014;59:468-473.

27. Gandia M, Morales-Espinoza EM, Martin-Gonzalez RM, et al. Factors influencing dry mouth in patients with primary Sjogren syndrome: usefulness of the ESSPRI index. Oral Health Dent Manag 2014;13:402-407.

28. Ayaki M, Kawashima M, Negishi K, Kishimoto T, Mimura $\mathrm{M}$, Tsubota K. Sleep and mood disorders in dry eye disease and allied irritating ocular diseases. Sci Rep 2016;6:22480.

29. Lee YB, Koh JW, Hyon JY, Wee WR, Kim JJ, Shin YJ. Sleep deprivation reduces tear secretion and impairs the tear film. Invest Ophthalmol Vis Sci 2014;55:3525-3531.

30. Boardman HF, Thomas E, Millson DS, Croft PR. The natural history of headache: predictors of onset and recovery. Cephalalgia 2006;26:1080-1088.

31. Karageorgas T, Fragioudaki S, Nezos A, Karaiskos D, Moutsopoulos HM, Mavragani CP. Fatigue in primary Sjogren's syndrome: clinical, laboratory, psychometric, and biologic associations. Arthritis Care Res (Hoboken) 2016;68:123-131.

32. Segal B, Thomas W, Rogers T, et al. Prevalence, severity, and predictors of fatigue in subjects with primary Sjogren's syndrome. Arthritis Rheum 2008;59:1780-1787.

33. Cho HJ, Yoo JJ, Yun CY, et al. The EULAR Sjogren's syndrome patient reported index as an independent determinant of health-related quality of life in primary Sjogren's syndrome patients: in comparison with non-Sjogren's sicca patients. Rheumatology (Oxford) 2013;52:2208-2217.

34. Wu CH, Hsieh SC, Lee KL, Li KJ, Lu MC, Yu CL. Pilocarpine hydrochloride for the treatment of xerostomia 
in patients with Sjogren's syndrome in Taiwan: a double-blind, placebo-controlled trial. J Formos Med Assoc 2006;105:796-803.

35. Meiners PM, Arends S, Brouwer E, Spijkervet FK, Vissink A, Bootsma H. Responsiveness of disease activity indices ESSPRI and ESSDAI in patients with primary Sjogren's syndrome treated with rituximab. Ann Rheum Dis 2012;71:1297-1302.

36. Strand V, van Vollenhoven RF, Lee EB, et al. Tofacitinib or adalimumab versus placebo: patient-reported outcomes from a phase 3 study of active rheumatoid arthritis. Rheumatology (Oxford) 2016;55:1031-1041.
37. Lasselin J, Rehman JU, Akerstedt T, Lekander M, Axelsson J. Effect of long-term sleep restriction and subsequent recovery sleep on the diurnal rhythms of white blood cell subpopulations. Brain Behav Immun 2015;47:93-99.

38. Lange T, Perras B, Fehm HL, Born J. Sleep enhances the human antibody response to hepatitis A vaccination. Psychosom Med 2003;65:831-835.

39. Buysse DJ, Hall ML, Strollo PJ, et al. Relationships between the Pittsburgh Sleep Quality Index (PSQI), Epworth Sleepiness Scale (ESS), and clinical/polysomnographic measures in a community sample. J Clin Sleep Med 2008;4:563-571. 